PROCEEDINGS OF THE

AMERICAN MATHEMATICAL SOCIETY

Volume 126, Number 8, August 1998, Pages 2241-2247

S $0002-9939(98) 04769-8$

\title{
UNIFORM PRIMENESS OF THE JORDAN ALGEBRA OF SYMMETRIC OPERATORS
}

\author{
L. L. STACHÓ AND B. ZALAR
}

(Communicated by Palle E. T. Jorgensen)

\begin{abstract}
In this note we establish the best possible constant for the general lower estimate for the Jacobson - McCrimmon operator on the algebra of symmetric operators acting on a Hilbert space.
\end{abstract}

\section{INTRODUCTION}

In this paper we continue the investigation on the uniform primeness constant in Jordan Banach algebras. Jordan algebras were introduced as a possible algebraic foundation of quantum mechanics. In quantum mechanics selfadjoint operators on a Hilbert space are of special interest. They do not form an associative algebra, i.e. if $A, B$ are selfadjoint, then $A B$ is not neccessarily selfadjoint. This leads to the introduction of the Jordan product $A \circ B=\frac{1}{2}(A B+B A)$, which makes the space of selfadjoint operators on a Hilbert space into a nonassociative algebra.

In general, a vector space $(\mathcal{A}, \circ)$ equipped with the binary product $\circ$ is a Jordan algebra if $a \circ b=b \circ a$ and $((a \circ a) \circ b) \circ a=(a \circ a) \circ(b \circ a)$ hold for all $a, b \in \mathcal{A}$. A considerable supply (but not all!) of Jordan algebras is given by

Example 1.1. Let $\mathcal{B}$ be an associative algebra and $\mathcal{A} \subset \mathcal{B}$ a subspace closed for squaring, i.e. $a \in \mathcal{A}$ implies $a^{2} \in \mathcal{A}$. Then $(\mathcal{A}, \circ)$ is a Jordan algebra where, as above, $a_{1} \circ a_{2}=\frac{1}{2}\left(a_{1} a_{2}+a_{2} a_{1}\right)$.

Jordan algebras are not used so much in quantum mechanics anymore, but they have fruitful applications in various parts of mathematics such as geometry of bounded domains, analysis on symmetric cones, statistics etc. Some recommended books are [2]-[4], [6], [8], [9], [12], [13] and [15], [16]. For the purely algebraic theory we refer to [5], [7] and [11].

One of the most important operators in the Jordan theory is the Jacobson McCrimmon operator, defined by

$$
U_{a, b}(x)=(a \circ x) \circ b+(b \circ x) \circ a-(a \circ b) \circ x .
$$

Received by the editors January 19, 1996.

1991 Mathematics Subject Classification. Primary 46L70, 15A45, 15A60, 16W10, 17 C65.

Key words and phrases. Hilbert space, bounded operators, symmetric operators, Jordan algebra, Jacobson-McCrimmon operator, prime algebra.

(C)1998 American Mathematical Society 
In the case of Jordan algebras from Example 1.1 this reduces to $U_{a, b}(x)=$ $\frac{1}{2}(a x b+b x a)$. According to the work of Beidar, Mikhalev and Slinko this operator also represents primeness of Jordan algebras, namely a nondegenerate $\mathcal{A}$ is prime if and only if $U_{a, b}=0$ implies $a=0$ or $b=0$.

In Banach Jordan algebras it is interesting to know whether there exists a uniform lower estimate for the operator $U_{a, b}$. Good estimates, we hope, can be used also in the theory of bounded symmetric domains.

In order to simplify further exposition we shall suppress the factor $\frac{1}{2}$, and so for the purpose of this paper we state

Definition 1.2. Let $\mathcal{A}$ be a Jordan algebra from Example 1.1. For $a, b, \in \mathcal{A}$ define $U_{a, b}: \mathcal{A} \rightarrow \mathcal{A}$ by

$$
U_{a, b}(x)=a x b+b x a .
$$

It seems that there exist three previous results in the direction of our work. Mathieu [10] proved that for prime $C^{*}$-algebras we have the lower estimate $\left\|U_{a, b}\right\| \geq$ $\frac{2}{3}\|a\| \cdot\|b\|$. Cabrera and Rodriguez [1] proved that for prime $J B^{*}$-algebras we have the lower estimate $\left\|U_{a, b}\right\| \geq \frac{1}{10206}\|a\| \cdot\|b\|$. Stachó and Zalar [14] proved that for standard operator algebras on a Hilbert space we have $\left\|U_{a, b}\right\| \geq 2(\sqrt{2}-1)\|a\| \cdot\|b\|$. Note that the upper estimate $\left\|U_{a, b}\right\| \leq 2\|a\| \cdot\|b\|$ is trivial.

In the present paper we are interested in the "quantum mechanical" Jordan algebra $\operatorname{Symm}(\mathcal{H})=\left\{A \in B(\mathcal{H}) ; A^{*}=A\right\}$. Here $\mathcal{H}$ denotes a Hilbert space, $B(\mathcal{H})$ the algebra of bounded operators acting on $\mathcal{H}$ and $A^{*}$ the usual adjoint of an operator $A$. In the sequel we prove

Theorem 1.3. Let $A, B \in \operatorname{Symm}(\mathcal{H})$. Then

$$
\left\|U_{A, B}\right\| \geq\|A\| \cdot\|B\| .
$$

Remark. The essential problem here is to compute

$$
\sup _{X=X^{*},\|X\|=1}\|A X B+B X A\|
$$

for $A, B$ selfadjoint, as opposed to

$$
\sup _{\|X\|=1}\|A X B+B X A\|,
$$

so we cannot use any of the existing results. The above estimate is, in general, the best possible.

\section{The TWO-Dimensional CASE}

By $\mathcal{M}_{2}$ we denote the algebra of $2 \times 2$ complex matrices equipped with the operator norm from $B\left(\mathbb{C}^{2}\right)$, where $\mathbb{C}^{2}$ is considered as a Hilbert space. We stress again that $U_{A, B}: \operatorname{Symm}\left(\mathbb{C}^{2}\right) \rightarrow \operatorname{Symm}\left(\mathbb{C}^{2}\right)$.

We can restrict our consideration to real matrices.

Lemma 2.1. We have

$$
\min _{\substack{A, B \in \operatorname{Symm}\left(\mathbb{C}^{2}\right) \\\|A\|=\|B\|=1}}\left\|U_{A, B}\right\| \geq \min _{\substack{A, B \in \operatorname{Symm}\left(\mathbb{R}^{2}\right) \\\|A\|=\|B\|=1}} \max _{\substack{X \in \operatorname{Symm}\left(\mathbb{R}^{2}\right) \\\|X\|=1}}\left\|U_{A, B}(X)\right\| .
$$


Proof. Given two complex symmetric matrices $A$ and $B$, there exists an orthonormal basis of $\mathbb{C}^{2}$ with respect to which the corresponding operators $v \mapsto A v, v \mapsto B v$ are both represented with real matrices. One such basis can be constructed from eigenvectors of $A$ which we denote by $x, y$. Since $A$ is symmetric, $x$ and $y$ are orthogonal. Given any $\varphi \in[0,2 \pi]$, the matrix representing $A$ in the basis $\left\{x, e^{i \varphi} y\right\}$ has real entries. $B$ is then represented by numbers $\langle B x, x\rangle,\langle B y, y\rangle$ and $e^{i \varphi}\langle B y, x\rangle$. The first two are real because $B$ is symmetric, while the last one can be made real by choosing an appropriate $\varphi$.

From here until Proposition 2.7 we assume that $A, B$ are symmetric norm-one $2 \times 2$ matrices with real entries. Denote

$$
V_{\varphi}=\left[\begin{array}{cc}
\cos \varphi & \sin \varphi \\
\sin \varphi & -\cos \varphi
\end{array}\right], \quad v_{\varphi}=\left[\begin{array}{c}
\cos \varphi \\
\sin \varphi
\end{array}\right] .
$$

Then $V_{\varphi}$ is a selfadjoint idempotent with norm one, and $v_{\varphi / 2}$ is an eigenvector with eigenvalue 1 of $V_{\varphi}$.

Since $A, B$ are selfadjoint and of norm one, both have 1 or -1 in their spectrum. The norm of $U_{A, B}$ does not change if we replace $A$ by $-A$ or if we switch the roles of $A$ and $B$. This leads to the following.

Observation 2.2. We may assume that $\sigma(A)=\{1, x\}$ and $\sigma(B)=\{1, y\}$, where $|x| \leq|y| \leq 1, x, y \in \mathbb{R}$, and that there exist $\varphi, \vartheta \in[0,2 \pi]$ such that

$$
A=V_{\varphi}\left[\begin{array}{ll}
1 & 0 \\
0 & x
\end{array}\right] V_{\varphi} \quad \text { and } \quad B=V_{\vartheta}\left[\begin{array}{ll}
1 & 0 \\
0 & y
\end{array}\right] V_{\vartheta}
$$

Lemma 2.3. (a) Let $a, b$ be real numbers. Then

$$
\max _{\varphi}|a \sin \varphi+b \cos \varphi|=\sqrt{a^{2}+b^{2}} .
$$

(b) Let $f(\lambda)=\lambda^{2}-t \lambda+d$ be a real polynomial. Then its roots lie in $[-1,1]$ if and only if $|t|-d \leq 1$.

Proof. Freshman calculus.

Proposition 2.4. Denote

$$
C=\left[\begin{array}{cc}
2 \cos \eta & (1+x y) \sin \eta \\
(1+x y) \sin \eta & -2 x y \cos \eta
\end{array}\right] \text { and } D=\left[\begin{array}{cc}
2 y \sin \eta & -(x+y) \cos \eta \\
-(x+y) \cos \eta & -2 x \sin \eta
\end{array}\right] \text {, }
$$

where $\eta=\varphi-\vartheta$. Then

$$
\left\|U_{A, B}\right\| \geq \max _{\delta}\|\cos \delta \cdot C+\sin \delta \cdot D\|,
$$

which further implies

(i) $\left\|U_{A, B}\right\| \geq \sqrt{4 \cos ^{2} \eta+(1+x y) \sin ^{2} \eta}$,

(ii) $\left\|U_{A, B}\right\| \geq 2 \sqrt{\cos ^{2} \eta+y^{2} \sin ^{2} \eta}$.

Proof. Define $\Phi: \mathcal{M}_{2}(\mathbb{R}) \rightarrow \mathcal{M}_{2}(\mathbb{R})$ by $\Phi(X)=V_{\varphi} X V_{\varphi}$. This $\Phi$ is an algebra isomorphism which is isometric. Hence $\Phi U_{A, B}=U_{\Phi A, \Phi B}$, and so

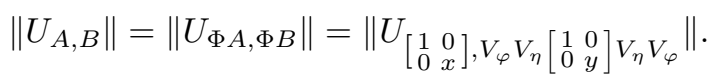

A straightforward computation shows that

$$
V_{\varphi} V_{\eta}\left[\begin{array}{ll}
1 & 0 \\
0 & y
\end{array}\right] V_{\eta} V_{\varphi}=V_{\varphi-\eta}\left[\begin{array}{ll}
1 & 0 \\
0 & y
\end{array}\right] V_{\varphi-\eta}
$$


Thus we may assume $A=\left[\begin{array}{ll}1 & 0 \\ 0 & x\end{array}\right]$ and $B=V_{\eta}\left[\begin{array}{ll}1 & 0 \\ 0 & y\end{array}\right] V_{\eta}$. Then, for any $\delta \in \mathbb{R}$, we have $\left\|U_{A, B}\right\| \geq\left\|U_{A, B}\left(V_{\eta-\delta}\right)\right\|$. However, a straightforward computation shows that

$$
\begin{aligned}
U_{A, B}\left(V_{\eta-\delta}\right) & =\left[\begin{array}{ll}
1 & 0 \\
0 & x
\end{array}\right] V_{\eta-\delta} V_{\eta}\left[\begin{array}{ll}
1 & 0 \\
0 & y
\end{array}\right] V_{\eta}+V_{\eta}\left[\begin{array}{ll}
1 & 0 \\
0 & y
\end{array}\right] V_{\eta} V_{\eta-\delta}\left[\begin{array}{ll}
1 & 0 \\
0 & x
\end{array}\right] \\
& =\cos \delta \cdot C+\sin \delta \cdot D .
\end{aligned}
$$

By specializing $\delta=0$, we obtain $\left\|U_{A, B}\right\| \geq\|C\|$. The norm of any matrix is not less than the norms of its rows and columns, and so $\left\|U_{A, B}\right\| \geq \sqrt{C_{11}^{2}+C_{12}^{2}}$ which is (i).

On the other hand, the upper left entry of $\cos \delta \cdot C+\sin \delta \cdot D$ is $\cos \delta \cdot c_{11}+\sin \delta \cdot d_{11}$. Since the operator norm of a matrix is bigger than the absolute value of any of its entries, we get $\left\|U_{A, B}\right\| \geq \max _{\delta}\left|C_{11} \cos \delta+D_{11} \sin \delta\right|$, which is, by Lemma 2.3(a), $\sqrt{C_{11}^{2}+D_{11}^{2}}$, and so (ii) is proved as well.

Corollary 2.5. If $x y \geq 0$, then $\left\|U_{A, B}\right\| \geq 1$.

Proof. By Proposition 2.4(i) we have

$$
\left\|U_{A, B}\right\|^{2} \geq(1+x y)\left\{\cos ^{2} \eta+\sin ^{2} \eta\right\}=1+x y \geq 1 .
$$

Now we write

$$
c=|\cos \eta| \quad \text { and } \quad p=|x y|
$$

in order to simplify the notations in further statements.

Proposition 2.6. Suppose that $x y \leq 0$ and $\left\|U_{A, B}\right\| \leq 1$. Then

(i) $p \leq \frac{1}{4}$,

(ii) $2(1+p) c-4 p c^{2}+(1-p)^{2}\left(1-c^{2}\right) \leq 1$,

(iii) $p \leq(1-p) c$.

Proof. By Proposition 2.4(ii), we have $1 \geq 2|y|$ and so $|y| \leq \frac{1}{2}$. We assumed that $|x| \leq|y|$; hence $p \leq \frac{1}{4}$. Since $1 \geq\left\|U_{A, B}\right\| \geq\|C\|$ and $\|C\|$ is the maximum of the absolute values of the roots of the characteristic polynomial $f(\lambda)=\lambda^{2}-\operatorname{trace}(C) \lambda+$ $\operatorname{det}(C)$, we have by Lemma 2.3 that $|\operatorname{trace}(C)|-\operatorname{det}(C) \leq 1$, which yields (ii).

For (iii), we consider the unit vector $v=\left[\begin{array}{l}\cos \frac{\eta}{2} \\ \sin \frac{\eta}{2}\end{array}\right]$, which is an eigenvector of $V_{\eta}$. Denote

$$
T=\left[\begin{array}{ll}
1 & 0 \\
0 & x
\end{array}\right] V_{\eta-\delta} V_{\eta}\left[\begin{array}{ll}
1 & 0 \\
0 & y
\end{array}\right] V_{\eta},
$$

where we use the facts which were established during the proof of Proposition 2.4. Then we have, because $T$ and $v$ have real entries,

$$
\begin{gathered}
\left\|U_{A, B}\right\| \geq\left\|T+T^{*}\right\| \geq\left|\left\langle\left(T+T^{*}\right) v, v\right\rangle\right|=2|\langle T v, v\rangle| \\
=2\left|\left\langle\left[\begin{array}{cc}
1 & 0 \\
0 & x
\end{array}\right]\left[\begin{array}{cc}
\cos \delta & \sin \delta \\
-\sin \delta & \cos \delta
\end{array}\right]\left[\begin{array}{ll}
1 & 0 \\
0 & y
\end{array}\right] V_{\eta} v, v\right\rangle\right| \\
=2\left|\left\langle\left[\begin{array}{cc}
\cos \delta & \sin \delta \\
-\sin \delta & \cos \delta
\end{array}\right]\left[\begin{array}{ll}
1 & 0 \\
0 & y
\end{array}\right] v,\left[\begin{array}{ll}
1 & 0 \\
0 & x
\end{array}\right] v\right\rangle\right| \\
=2|a \cos \delta+b \sin \delta|
\end{gathered}
$$


where $a=\frac{1-p}{2}+\frac{1+p}{2} \cos \eta$ and $b=\frac{y-x}{2} \sin \eta$. By Lemma 2.3, we have

$$
\left\|U_{A, B}\right\| \geq 2 \sqrt{a^{2}+b^{2}}=\sqrt{(1-p+(1+p) \cos \eta)^{2}+(y-x)^{2} \sin ^{2} \eta} .
$$

Now $(y-x)^{2}=y^{2}+x^{2}-2 x y=y^{2}+x^{2}+2|x y| \geq 4|x y|=4 p$, and so, since $1-p$ is positive,

$$
\begin{aligned}
1 & \geq\left\|U_{A, B}\right\|^{2} \geq(1-p-(1+p) \cos \eta)^{2}+4 p\left(1-c^{2}\right) \\
& \geq(1-p)^{2}-2 c(1-p)(1+p)+(1+p)^{2} c^{2}+4 p-4 p c^{2} \\
& =(1+p)^{2}-2 c(1-p)(1+p)+(1+p)^{2} c^{2}=(1+p-c(1-p))^{2},
\end{aligned}
$$

which completes the proof.

Proposition 2.7. Let $A, B$ be symmetric real two-by-two matrices with norm 1 . Then there exists $V$ with the same properties such that $\|A V B+B V A\| \geq 1$.

Proof. Assume that this is not true and so $\left\|U_{A, B}\right\|<1$ should hold. By Corollary 2.5 , we have $x y<0$. By Proposition 2.6, we have

$$
\begin{gathered}
2(1+p) c+(1-p)^{2}-(1+p)^{2} c^{2} \leq 1, \\
(1-p)^{2} \leq(1-(1+p) c)^{2} .
\end{gathered}
$$

The possibility $1-p \leq 1-(1+p) c$ together with Proposition 2.6(iii) implies $p=0$, which contradicts Corollary 2.5. The second possibility $1-p \leq-1+(1+p) c$ implies (see Proposition 2.6(i))

$$
c \geq \min _{0 \leq p \leq 1 / 4} \frac{2-p}{1+p}>1
$$

which is again impossible.

Corollary 2.8. Let $A, B \in \operatorname{Symm}\left(\mathbb{C}^{2}\right)$. Then the estimate $\left\|U_{A, B}\right\| \geq\|A\| \cdot\|B\|$ holds.

Proof. Because of Lemma 2.1 and Observation 2.2 it is sufficient to establish that $\max _{\delta}\left\|U_{A, B}\left(V_{\delta}\right)\right\| \geq 1$ for symmetric real matrices of the form $A=\left[\begin{array}{ll}1 & 0 \\ 0 & x\end{array}\right], B=$ $V_{\eta}\left[\begin{array}{ll}1 & 0 \\ 0 & y\end{array}\right] V_{\eta}$, where $|x| \leq|y| \leq 1$. This is done in the previous proposition.

\section{The General CASE}

In this section we complete the proof of Theorem 1.3 by a two-step reduction to the two-dimensional case.

Suppose first that $\mathcal{H}$ is finite-dimensional and $A, B \in \operatorname{Symm}(\mathcal{H})$. Without loss of generality we may assume that $\|A\|=\|B\|=1$ and $1 \in \sigma(A) \cap \sigma(B)$. Thus there exist unit vestors $\xi, \eta \in \mathcal{H}$ such that $A \xi=\xi$ and $B \eta=\eta$. Let $\mathcal{K}$ be the span of $\{\xi, \eta\}$. If $\mathcal{K}$ is one-dimensional, then $\left\|U_{A, B}\right\| \geq 1$ is obvious. If $\mathcal{K}$ is twodimensional, then let $P: \mathcal{H} \rightarrow \mathcal{H}$ be the orthogonal (selfadjoint) projection onto $\mathcal{K}$. Define $\phi: \operatorname{Symm}(\mathcal{H}) \rightarrow \operatorname{Symm}(\mathcal{H})$ by $\phi(X)=P X P$. It is obvious that $\|\phi\| \leq 1$ and that $\operatorname{ran}(\phi)$ is isometrically isomorphic (as a Jordan algebra) to $\operatorname{Symm}\left(\mathbb{C}^{2}\right)$. 
Since $P \xi=\xi$ and $P \eta=\eta$, we have $\|\phi(A)\|=\|\phi(B)\|=1$. Moreover

$$
\begin{aligned}
\left\|U_{A, B}\right\| & =\sup _{\|X\|=1, X=X^{*}}\|A X B+B X A\| \\
& \geq \sup _{\|X\|=1, X=X^{*}}\|P A X B P+P B X A P\| \\
& \geq \sup _{Y=Y^{*},\|P Y P\|=1} \| P A P Y P B P+P B P Y P A P \mid \\
& =\sup _{Z \in \operatorname{Im}(\phi),\|Z\|=1}\left\|U_{\phi(A), \phi(B)}(Z)\right\| \\
& \geq\left\|U_{\phi(A), \phi(B)}^{\prime}\right\| \geq\|\phi(A)\| \cdot\|\phi(B)\|=1
\end{aligned}
$$

where $U^{\prime}:(\operatorname{Im} \phi) \rightarrow(\operatorname{Im} \phi)$ and Corollary 2.8 was used.

Finally take $\mathcal{H}$ to be infinite-dimensional and let $A, B \in \operatorname{Symm}(\mathcal{H})$ be normone operators. Let a positive $\epsilon$ be given. There exist unit vectors $\xi, \eta \in \mathcal{H}$ such that $\|A \xi\|,\|B \eta\| \geq 1-\epsilon$. Let $\mathcal{K}$ denote the span of $\{\xi, \eta, A \xi, B \eta\}$ and $P: \mathcal{H} \rightarrow$ $\mathcal{H}$ the orthogonal projection onto $\mathcal{K}$. Define again $\phi(X)=P X P$ which makes $\operatorname{Im}(\phi)$ isometrically isomorphic (as a Jordan algebra) to $\operatorname{Symm}(\mathcal{K})$, which is finitedimensional. We have $\|\phi(A)\|,\|\phi(B)\|>1-\epsilon$, and by the above paragraph we have, using the same type of argument,

$$
\left\|U_{A, B}\right\| \geq(1-\varepsilon)^{2}
$$

By letting $\varepsilon \rightarrow 0$ we finish the proof of Theorem 1.3.

Remark 3.1. The estimate given in Theorem 1.3 is, in general, the best possible. Indeed, if $\mathcal{H}$ is at least two-dimensional, we can take orthogonal unit vectors $\alpha, \beta$ and form $P, Q \in \operatorname{Symm}(\mathcal{H})$ by $P \xi=\langle\xi, \alpha\rangle \alpha, Q \xi=\langle\xi, \beta\rangle \beta$. It is easy to check that $\left\|U_{P, Q}\right\|=1$.

Problems. It is still unclear how to compute the precise value of

$$
\min _{\|A\|=\|B\|=1}\left\|U_{A, B}\right\|
$$

in von Neumann factors of type II and type III, or even how to give some useful estimate in the form $1+f(A, B) \leq\left\|U_{A, B}\right\| \leq 1+g(A, B)$. In many cases $\left\|U_{A, B}\right\| \leq$ $1+\|A B\|$ but we do not know whether this holds in general.

\section{ACKNOWLEDGMENT}

A part of this research was done during the second author's visit at London and Reading, which was supported by the British Council of Slovenija. He thanks Cho-Ho Chu and Les Bunce for hospitality and stimulating conversations.

\section{REFERENCES}

1. M. Cabrera Garcia and A. Rodriguez-Palacios, Non-degenerately ultraprime Jordan-Banach algebras: a Zelmanovian treatment, Proc. London Math. Soc. 69 (1994), 576-604. MR 95g:46094

2. S. Dineen, The Schwarz Lemma, Oxford Science Publications, 1989. MR 91f:46064

3. J. Faraut and A. Koranyi, Analysis on Symmetric Cones, Oxford Press, 1994. CMP 97:12

4. J.M. Isidro and L.L. Stachó, Holomorphic Automorphism Groups in Banach Spaces: An Elementary Introduction, North-Holland, 1985. MR 86f:32037

5. N. Jacobson, Structure and Representations of Jordan algebras, Amer. Math. Soc., 1968. MR 40:4330

6. Jordan Algebras (W. Kaup, K. McCrimmon and H.P. Petersson, eds.), Walter de Gruyter, 1994. MR 95c: 17002 
7. O. Loos, Jordan Pairs, Lecture Notes in Math., vol. 460, Springer Verlag, 1975. MR 56:3071

8. O. Loos, Bounded Symmetric Domains and Jordan Pairs, University of California at Irvine, 1977.

9. J.D. Malley, Statistical applications of Jordan algebras, Lecture Notes in Statistics, vol. 91, Springer-Verlag, 1994. MR 96i:62078

10. M. Mathieu, More properties of the product of two derivations of a $C^{*}$-algebra, Bull. Austral. Math. Soc. 42 (1990), 115-120. MR 91k:46079

11. E. Neher, Jordan Triple Systems by the Grid Approach, Lecture Notes in Math., vol. 1280, Springer Verlag, 1987. MR 89b:17024

12. I. Satake, Algebraic Structures of Symmetric Domains, Iwanami Shoten, Tokyo, and Princeton Univ. Press., Princeton, NJ, 1980. MR 82i:32003

13. T.A. Springer, Jordan Algebras and Algebraic Groups, Springer Verlag, 1973. MR 52:523

14. L.L. Stachó and B. Zalar, On the norm of Jordan elementary operators in standard operator algebras, Publ. Math. Debrecen 49 (1996), 127-134. MR 97k:47043

15. H. Upmeier, Symmetric Banach Manifolds and Jordan $C^{*}$-algebras, North-Holland, 1985. MR 87a:58022

16. H. Upmeier, Jordan Algebras in Analysis, Operator Theory and Quantum Mechanics, CBMS Regional Conf. Ser. Math., vol. 67, American Mathematical Society, 1987. MR 88h:17032

Bolyai Intézet, Aradi Vértanuk tere 1, 6720 Szeged, Hungary

E-mail address: stacho@math.u-szeged.hu

University of Maribor, Faculty of Civil Engineering, Department of Basic Sciences, Smetanova 17, 62000 Maribor, SloveniJa

E-mail address: borut.zalar@uni-mb.si or borut.zalar@uni-lj.si 